\title{
Dalteparin Sodium
}

National Cancer Institute

\section{Source}

National Cancer Institute. Dalteparin Sodium. NCI Thesaurus. Code C75767.

The sodium salt of a low molecular weight, synthetic heparin. As an

anticoagulant/antithrombotic agent, dalteparin binds to antithrombin and enhances the inhibition of Factor Xa. Compared to unfractionated heparins, the use of dalteparin is associated with lower incidences of osteoporosis and heparin-induced thrombocytopenia. 